\title{
Recubrimiento comestible natural con base en Aloe vera como estrategia de conservación de Psidium guajava
}

\author{
Natural edible coating based on Aloe vera as a conservation strategy of Psidium \\ guajava
}

\section{Revestimento comestível natural à base de Aloe vera como uma estratégia de conservação de Psidium guajava}

\author{
George Adalberto García-Mera ${ }^{1}$ \\ Carlos Alfredo Salas-Macías ${ }^{2}$ \\ Heydi Gwendoline Canales-Torres ${ }^{3}$
}

Recibido: mayo de 2017

Aceptado: agosto de 2017

Para citar este artículo: García-Mera, G.A., Salas-Macías, C.A., y Canales-Torres, H,G. (2017). Recubrimiento comestible natural con base en Aloe vera como estrategia de conservación de Psidium guajava. Revista Científica, 30 (3), 224-236. Doi: https://doi.org/10.14483/23448350.11790

\section{Resumen}

La industria alimenticia a nivel mundial se encuentra en la necesidad de desarrollar nuevas estrategias tecnológicas y cambios para el favorecimiento de la preservación de las frutas. En este contexto, los recubrimientos han representado grandes beneficios para la industria y para el consumidor. La presente investigación tiene como objetivo principal evaluar el efecto de la aplicación de un recubrimiento natural utilizando como insumo principal el Aloe vera en guayaba, que por su naturaleza climatérica tiende a alcanzar la senescencia muy rápidamente en los procesos postcosecha, perdiendo calidad en muy corto tiempo. A fin de lograr el objetivo planteado se determinaron siete tratamientos para la evaluación de tres dosis de Aloe vera $(10 \%, 20 \%$ y $30 \%)$ mezclados con dos dosis de glicerol $(1.5 \%$ y $2.5 \%$ ). Como variables respuesta se consideraron: la pérdida de peso (porcentaje de pérdida con respecto al peso de fruta fresca), el $\mathrm{pH}$ de las frutas, sólidos solubles totales (SST), acidez titulable y la presencia de microorganismos por medio del total de unidades formadoras de colonias (UFC). El mejor tratamiento fue el T5, ya que contaba con los mejores valores en cada una de las variables analizadas. Dicho tratamiento estuvo compuesto por $30 \%$ de Aloe vera y $1.5 \%$ de glicerol. Luego de la evaluación se concluye que el recubrimiento aplicado en guayaba con base en Aloe vera y glicerol logra retardar el tiempo de maduración en la guayaba conservándola y logrando mantener a la fruta en buen estado conforme al paso del tiempo, sin perder sus características organolépticas.

Palabras clave: industria alimentaria, tecnología alimentaria, procesamiento de alimentos, maduración, películas comestibles.

Facultad de Ciencias Agropecuarias. Universidad Laica "Eloy Alfaro de Manabí. Manta, Ecuador. Contacto: georgarcia@uleam.edu.ec Facultad de Ingeniería Agronómica. Universidad Técnica de Manabí. Km 15 via a Lodana, Santa Ana. Ecuador. Contacto: csalas@utm.edu.ec Umbrella EcoConsulting S.A.C. Perú. Contacto: hcanales@ue.com.pe 


\begin{abstract}
Global food industry encounters the need for new strategies and technological changes that favor the preservation of fruits. In this sense, coatings have shown great benefits for the industry and consumers. This research has as main objective to evaluate the effect of the application of a natural coating using as a main ingredient Aloe vera in guava, which by its climacteric nature tends to reach senescence quickly in the post-harvest processes, losing quality in very short time. In order to achieve the stated objective, seven treatments to evaluate three doses of Aloe vera $(10 \%, 20 \%$ and $30 \%)$ mixed with two doses of glycerol $(1.5 \%$ and $2.5 \%)$ were determined. The response variables were: weight loss (loss percentage relative to the weight of fresh fruit), $\mathrm{pH}$ of the fruits, total soluble solids (TSS), titratable acidity, presence pathogens defined by total colony forming units (CFU). The best treatment was T5, due to it had the best values in each of the variables analyzed. Such treatment consisted of $30 \%$ Aloe vera and $1.5 \%$ glycerol. The evaluation concludes that the coating based on Aloe vera and glycerin applied to guava, is able to reduce maturation and managing guava fruit to keep in good condition according to the passage of time.
\end{abstract}

Keywords: Food industry, food technology, food processing, maturation, edible films.

\section{Resumo}

A indústria global de alimentos atende a necessidade de novas estratégias e mudanças tecnológicas que favoreçam a preservação de frutas. Neste contexto, os revestimentos têm mostrado grandes benefícios para a indústria e para os consumidores. Esta pesquisa tem como objetivo principal avaliar o efeito da aplicação de um revestimento natural, utilizando como principal ingrediente Aloe vera em goiaba, que por sua natureza climatérica tende a alcançar a senescência rapidamente nos processos de pós-colheita, perdendo qualidade em pocou tempo. A fim de atingir os objectivos declarados, foram determinados sete tratamentos para avaliar três doses de Aloé vera $(10 \%, 20 \%$ e $30 \%)$ misturados com duas doses de glicerol (1.5\% e $2.5 \%$ ). Como variáveis de resposta foram consideradas: perda de peso (perda percentual em relação ao peso de frutas frescas), o pH dos frutos, sólidos solúveis totais (SST), acidez titulável, presença de patógenos definido pelo total de unidades formadoras de colónias (UFC). O melhor tratamento foi o T5, uma vez que tinha os melhores valores em cada uma das variáveis analisadas. Tal tratamento consistiu em $30 \%$ de Aloe vera e $1.5 \%$ de glicerol. A avaliação concluiu que o revestimento aplicado a goiaba, à base de Aloe vera e glicerina consegue diminuir o tempo de maturação em goiaba, mantendo a fruta em boas condições de acordo com a passagem de tempo sem perder suas características organolépticas .

Palavras-chaves: indústria de alimentos, tecnologia de alimentos, processamento de alimentos, maturação, filmes comestíveis.

\section{Introducción}

De manera tradicional, según el patrón de respiración, se ha determinado que las frutas se clasifican en climatéricas y no climatéricas (Giovannoni, 2001). En la primera, los frutos maduran debido al rápido incremento en la tasa de respiración, misma que generalmente se asocia con una elevada producción de etileno. Una vez iniciada la maduración climatérica la respiración llega un punto denominado máximo climatérico; luego de este punto la velocidad de respiración disminuye hasta el comienzo de la senescencia o muerte del fruto. Por tal motivo, la distribución comercial de un fruto climatérico se debe hacer antes de este máximo, de forma que termine de madurar fuera del árbol, evitando que se produzcan pérdidas de producto por manejo postcosecha (Pech et al., 2008).

El guayabo (Psidium guajava L.) es uno de los frutales climatéricos tropicales y subtropicales con amplia aceptación a nivel global (Gutiérrez et al., 2008) con un alto valor nutricional, que unido a lo rentable de su cultivo, ha posibilitado que adquiera gran importancia económica en varias regiones del mundo (Sanabria et al., 2005; Vasco et al., 2003, Lima et al., 2002). Su etapa de crecimiento es favorable, ya que puede darse en diversas condiciones climáticas, situación que ha dado paso a 
que sea introducida en muchos países (Salazar et al., 2006).

Recientemente, su producción comercial se ha difundido a diversas regiones y países, destacándose, entre otros: Estados Unidos, Australia, Filipinas, India, Sudáfrica, Venezuela, Brasil, Egipto, Tailandia e Indonesia (Aserca, 1996). En el Ecuador, se mencionan como las zonas de mayor producción a Santa Clara, Mera, Pastaza y Baños, ubicadas en las provincias de Pastaza y Tungurahua respectivamente.

Es un cultivo que tiene amplias perspectivas, ya que su uso y aprovechamiento puede incluir no solo el consumo como fruta fresca, sino también en diversos productos obtenidos mediante su transformación (jaleas, conservas, pastas, ates, bebidas enlatadas, etc.); por ello, el aumento de su producción presenta una oportunidad de diversificar su consumo (Yam Tzec et al., 2010). Otro factor de importancia, brevemente mencionado anteriormente, es su contenido nutricional, ya que contiene altas cantidades de ácido ascórbico, fósforo, calcio, hierro, tiamina, niacina y riboflavina (Jagtian, 2012).

Durante su ciclo de vida, la guayaba sufre muchos cambios en su composición. Estos son de relativa importancia para comprender los procesos metabólicos relacionados con el desarrollo, maduración y senescencia, desarrollo de óptimas cualidades sensoriales y aparición de síntomas relacionados con los fenómenos de pardeamiento o ablandamiento enzimático. Muchos de los cambios mencionados siguen su proceso una vez que los frutos son cosechados, dado que la actividad metabólica continúa variando de acuerdo al avance de la maduración (Bashir y Abu-Goukh, 2003), con la cual se relacionan directamente. Este hecho se debe a que los tejidos continúan respirando y los azucares, entre otros componentes, sufren modificaciones que culminan en la producción de $\mathrm{CO}_{2}$ y $\mathrm{H}_{2} \mathrm{O}$ (Gutiérrez et al., 2008).

Dentro de lo fenómenos que se presentan durante la maduración y senescencia se podía citar la respiración, endulzamiento, cambio en la textura y aroma, producción de compuestos volátiles, cambios en la coloración, disminución del valor nutritivo, entre otros. Fenómenos que varían según la especie y estado de madurez del mismo, no obstante, se ha llegado a comprobar que también se producen diferenciaciones dentro de la misma variedad (Bashir y Abu-Goukh, 2003). Dichos fenómenos producen en la fruta pérdidas de peso y deterioro desmejorando su apariencia y calidad (Saenz et al., 1991), hecho que se observan con mayor frecuencia en la cadena de comercialización y aumenta debido al manejo inadecuado que recibe el producto (Martínez et al., 2005).

Por lo expuesto, es comprensible esperar una afectación directa en la demanda del producto y las preferencias del consumidor. Por ello, que se presenta la necesidad de buscar alternativas que permitan aumentar el tiempo y calidad de preservación de los frutos por medio de sistemas de empaque que consideren la actividad respiratoria postcosecha (De la Cruz et al., 1998). Dentro de este campo se encuentran los recubrimientos, los cuales disminuyen parcialmente el proceso respiratorio y retardan la fase de maduración, sin afectar las características organolépticas y propiedades nutricionales, presentando así grandes beneficios para la industria de alimentos y para el consumidor (Bastioli, 2001).

Este método de conservación, además de actuar como un sistema de empaque durante el almacenamiento, retarda el deterioro, realza atributos de calidad y puede actuar frente a los microorganismos cuando se incorporan compuestos antimicrobianos que impiden su crecimiento y desarrollo (Petersen et al., 1999). De igual manera, permiten la incorporación de antioxidantes, sales de calcio o ingredientes funcionales como minerales y vitaminas (Oms-Oliu et al., 2010), o el empleo de biopolímeros y algunas proteínas de fuentes naturales (Famá et al., 2007; Cuq et al., 1998) disponibles en el mercado y que son ampliamente utilizados para mejorar la calidad.

En adición a lo expuesto, esta técnica de almacenamiento ayuda a modificar la composición 
de gases que rodean la fruta, elevando la concentración de $\mathrm{CO}_{2}$ y disminuyendo el $\mathrm{O}_{2}$ disponible para la respiración, permitiendo un intercambio gaseoso controlado (Sundaresan y Ludescher, 2008). Esto con la finalidad de disminuir la velocidad metabólica y humedad (Ramírez et al., 2013), creando condiciones adecuadas para el almacenamiento amplio al producto (Sarroca y Torres, 2006; Kader et al., 1989; Mannapperuma et al., 1989; Hintlian y Hotchkiss, 1986).

La presente investigación tiene como objetivo principal evaluar los efectos de la aplicación de un recubrimiento natural utilizando como insumo principal el Aloe vera en frutas de guayaba, que por su naturaleza climatérica tiende a alcanzar la senescencia muy rápidamente en los procesos postcosecha, perdiendo calidad en muy corto tiempo. De igual manera, la investigación trata de aportar nuevos conocimientos sobre técnicas de conservación de frutas con el fin de que la industria alimenticia pueda brindar inocuidad, calidad, conservación de sus características organolépticas y a la vez minimizar las pérdidas económicas en la comercialización de las mismas.

\section{Materiales y métodos}

El presente trabajo se realizó en los laboratorios de procesos y en el de microbiología de la Facultad de Ciencias Agropecuarias de la Universidad Laica Eloy Alfaro de Manabí, de la Ciudad de Manta, Ecuador.

Los procesos metodológicos utilizados para la presente investigación (figura 1) se detallan en el diagrama siguiente:

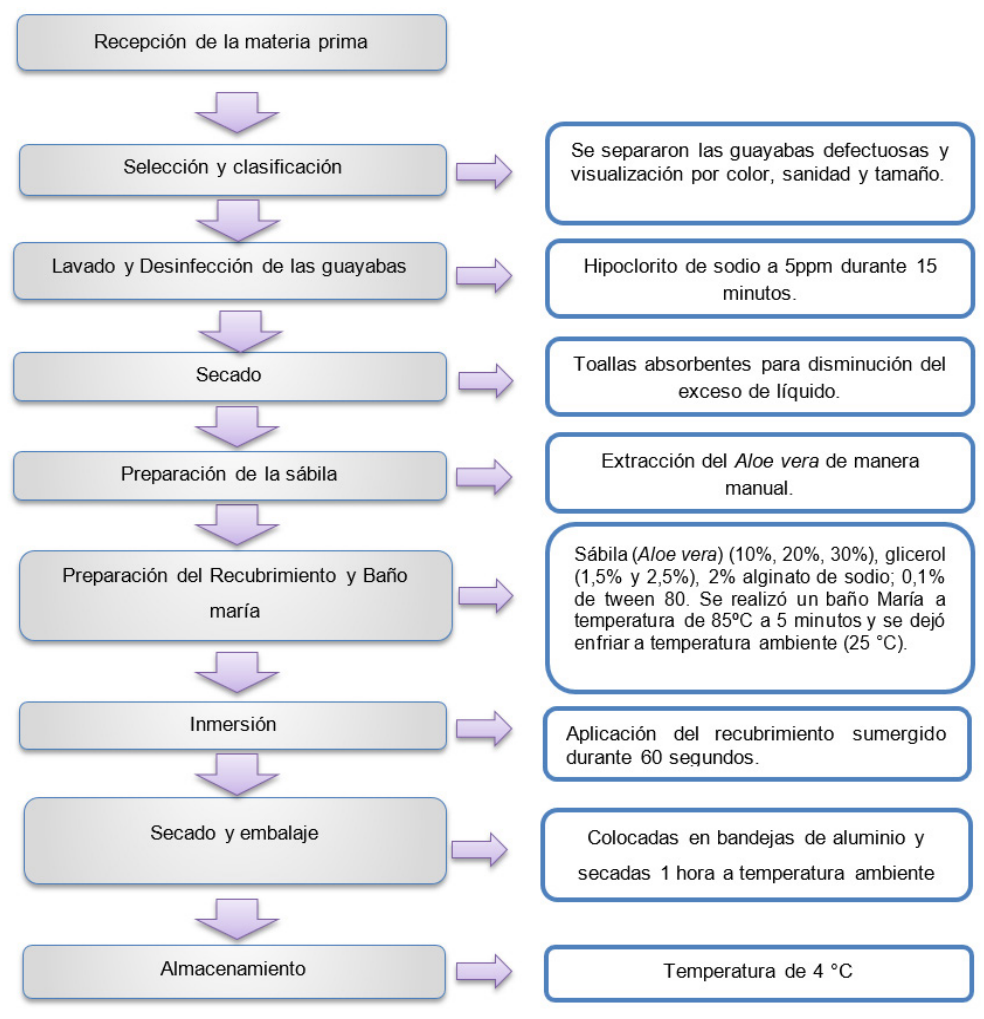

Figura 16. Diagrama de proceso metodológico para determinar los efectos de un recubrimiento comestible natural con base en sábila (Aloe vera) como estrategia de conservación de la guayaba (Psidium guajava L).

Fuente: elaboración propia. 
Tabla 1. Tratamientos para determinar el efecto de un recubrimiento comestible natural con base en sábila

(Aloe vera) como estrategia de conservación de la guayaba (Psidium guajava L.)

\begin{tabular}{cccc}
\hline \multicolumn{3}{c}{ Factores en estudio } \\
Tratamientos & Código & $\begin{array}{c}\text { \% Solución de } \\
\text { sábila } \\
\text { (Aloe vera) }\end{array}$ & \% Glicerol \\
& & $10 \%$ & $1.5 \%$ \\
1 & A1B1 & $10 \%$ & $2.5 \%$ \\
2 & A1B2 & $20 \%$ & $1.5 \%$ \\
3 & A2B1 & $20 \%$ & $2.5 \%$ \\
4 & A2B2 & $30 \%$ & $1.5 \%$ \\
5 & A3B1 & $30 \%$ & $2.5 \%$ \\
6 & A3B2 & $0 \%$ & $0 \%$ \\
7 & Testigo & & \\
\hline
\end{tabular}

Fuente: elaboración propia.

Las variables respuesta para determinar el efecto del recubrimiento sobre las guayabas, fueron:

- Pérdida de peso. Se llevó a cabo un seguimiento de la pérdida del peso del fruto en cada uno de los tratamientos durante su almacenamiento $(0,3,6,9,12$ y 15 días $)$ con una balanza digital a temperatura ambiente $\left(25^{\circ} \mathrm{C}\right)$. Los resultados se expresaron como porcentaje de peso perdido basado en el peso inicial y final del fruto, considerando como pérdida de peso durante cada periodo de almacenamiento con base a peso fresco del fruto.

- Ph. Se determinó a través de un potenciómetro portátil tomando $10 \mathrm{gr}$ de muestra en un vaso de precipitación de $50 \mathrm{ml}$. La muestra fue filtrada utilizando una gaza a manera de tamiz y el resultado se colocó en un matraz Erlenmeyer de $250 \mathrm{ml}$. Posteriormente, se introdujo el electrodo del potenciómetro en la muestra hasta aparézcala aparición de la lectura en la pantalla del potenciómetro. Se tomaron tres lecturas para cada tratamiento, para luego calcular un promedio de cada uno de ellos.

- Solidos solubles totales. Se evaluaron tres frutos de cada tratamiento durante los días de muestreo $(0,3,6,9,12$ y 15 días). Las muestras se molieron en un procesador durante tres minutos. El contenido de sólidos solubles totales (SST) de la muestra se evaluó empleando un refractómetro. Una gota de la muestra fue colocada en el prisma del refractómetro de manera directa tomando tres muestras de cada tratamiento. El contenido de SST se expresó como porcentaje de la escala ${ }^{\circ}$ Brix.

- Acidez titulable. Para la determinación de la acidez titulable se tomaron $10 \mathrm{ml}$ de muestra y se le adicionó $20 \mathrm{ml}$ de agua destilada para diluir. Posteriormente se realizó la titulación con hidróxido de sodio $(\mathrm{NaOH} 0.01 \mathrm{~N})$ a $\mathrm{pH}$ de 8.3 utilizando como indicador la fenolftaleína; este procedimiento se realizó por triplicado para cada tratamiento por día de muestreo. La determinación de ácido cítrico se utilizó la ecuación

$$
\text { Ácido cítrico }(\%)=\frac{\mathrm{V} \times \mathrm{N} \times \mathrm{Meq}}{\text { Alícuota valorada }} \times 100
$$

Dónde:

$\mathrm{V}=$ volumen de $\mathrm{NaOH}$ gastados $(\mathrm{ml})$.

$\mathrm{N}=$ normalidad del $\mathrm{NaOH}$.

Meq = miliequivalente del ácido que se encuentra en mayor proporción de la muestra (0.064 para ácido cítrico).

Alícuota valorada $=$ peso en gr, o volumen de muestra en ml.

- Presencia de mohos y levaduras. Se llevó a cabo un análisis microbiológico para determinar la presencia de unidades formadoras de colonias (UFC) en el laboratorio de la Facultad de Ciencias Agropecuarias de la Universidad Laica Eloy Alfaro de Manabí (Uleam).

Se utilizó un diseño completamente al azar (DCA) con arreglo bifactorial $\left(A^{*} B\right)$, con tres repeticiones para completar 21 unidades experimentales. El análisis estadístico se realizó con el software Infostat. De igual manera, se realizaron comparaciones de medias de tukey al 5\%. 


\section{Resultados y discusión}

\section{Pérdida de peso}

La tabla 2 presenta los resultados para la variable respuesta pérdida de peso. El análisis de varianza demuestra diferencias significativas entre los tratamientos en estudio evidenciándose la influencia de la sábila (Aloe vera) y el glicerol en la pérdida de peso. De igual manera, $\mathrm{Ni}$ et al. (2004) mencionan que el Aloe vera, siendo su composición básicamente polisacáridos, fue efectivo frente a la pérdida de humedad sin incorporación lipídica, al igual que con otros recubrimientos comestibles (Perez-Gago et al., 2005).

En cuanto al test de Tukey al $(p<0.05)$ para la pérdida de peso, se determinó que el tratamiento con mayor pérdida fue T7 o testigo, Ilegando a tener hasta un $6.83 \%$ de pérdida en producto no recubierto, promediando un $4.38 \%$, situación similar a resultados presentados por Castellano et al. (2005) y Tomás et al. (2005) en frutos sin recubiertas, diferenciándose con tratamientos recubiertos, debido a que se logró aminorar la transpiración. Las menores pérdidas se dieron en el tratamiento T5 que tuvo hasta un $2.13 \%$ en pérdidas promediando un $1.86 \%$. Este hecho demuestra que la mayor concentración de sábila (Aloe vera) y menor concentración de glicerol influyen disminuyendo la pérdida de agua en la fruta, logrando aminorar las pérdidas de peso en las guayabas.

Por lo expuesto, estas pérdidas de peso en el fruto se deben a que posiblemente al intercambio de gases durante el proceso de respiración y transpiración que disminuyen el contenido de agua (Gómez et al., 2002) que, de manera general en frutas, se establece un máximo permisible de aproximadamente un 5\% (Cisternas, 1996) y por encima del $10 \%$ para el deterioro de las mismas (Espinoza, 2015). Con respecto a este punto, es necesario indicar que uno de los principales propósitos de la aplicación de un recubrimiento sobre la superficie de la guayaba es precisamente retardar la migración de humedad y la pérdida de compuestos volátiles.

La figura 2 presenta la pérdida de peso en cada uno de los tratamientos, considerando el tiempo de almacenamiento. Resultados similares se han encontrado en numerosas investigaciones (Achipiz et al., 2013; Zebadúa et al., 2005; Quila, 2003; Possel, 1992), donde se determina que la pérdida de peso se incrementa en la medida en que se incrementa el tiempo de almacenamiento.

Tabla 2. Pérdida de peso (\%) promedio de las muestras.

\begin{tabular}{|c|c|c|c|c|c|c|c|}
\hline \multirow{2}{*}{ Tratamientos } & \multicolumn{6}{|c|}{ Días } & \multirow{2}{*}{ Promedio } \\
\hline & 0 & 3 & 6 & 9 & 12 & 15 & \\
\hline T1 (A1B1) & 0 & 2.01 & 2.38 & 3.25 & 3.76 & 4.06 & 3.09 \\
\hline T2 (A1B2) & 0 & 2.24 & 2.51 & 3.30 & 3.55 & 4.24 & 3.17 \\
\hline T3 (A2B1) & 0 & 1.93 & 2.21 & 2.47 & 2.97 & 3.20 & 2.56 \\
\hline T4 (A2B2) & 0 & 2.23 & 2.48 & 2.91 & 3.08 & 3.67 & 2.87 \\
\hline T5 (A3B1) & 0 & 1.52 & 1.72 & 1.92 & 1.99 & 2.13 & 1.86 \\
\hline T6 (A3B2) & 0 & 1.95 & 2.02 & 2.52 & 2.68 & 2.97 & 2.43 \\
\hline T7 (Testigo) & 0 & 2.93 & 3.02 & 4.09 & 5.37 & 6.52 & 4.38 \\
\hline
\end{tabular}

Fuente: elaboración propia. 




Figura 2. Pérdidas de peso (\%) de frutos de guayaba durante el periodo de evaluación.

Fuente: elaboración propia.

$\mathrm{Ph}$

Las variaciones de $\mathrm{pH}$ son diferentes entre las especies. No obstante, se ha demostrado que existen diferencias en la misma especie debido a factores como la madurez, condiciones agronómicas y operaciones postcosecha a las que han sido sometidas. La tabla 3 presenta los valores de $\mathrm{pH}$ obtenidos durante la evaluación de los tratamientos en estudio y demuestra un aumento progresivo de este parámetro con respecto al tiempo de almacenamiento. El análisis de varianza presenta diferencias significativa entre los tratamientos donde la influencia del glicerol en las recubiertas mantuvo valores menores que en las no recubiertas (T7).

El test de comparación de medias de Tukey presentó diferencias entre los tratamientos motivo de estudio $(p<0,05)$. Siendo T7 el tratamiento con mayores valores registrados, Ilegando hasta un máximo de 4.27 comparados con los tratamientos con recubrimiento, los cuales mantenían $\mathrm{pH}$ relativamente bajos. El menor $\mathrm{pH}$ se encontró en el T5 con valores máximos de 3.83 al inicio del periodo de evaluación y un promedio de 3.77 , siendo el mejor tratamiento por su eficiencia para mantener el $\mathrm{pH}$ bajo. Al respecto, se debe indicar que el $\mathrm{pH}$ durante el almacenamiento demuestra la senescencia del fruto.

\section{Sólidos solubles totales de las guayabas}

Los sólidos solubles totales o ${ }^{\circ}$ Brix son aquellos que representan el porcentaje de sacarosa que hay en el jugo de la fruta, parámetro que indica la madurez de los frutos pues la cantidad normalmente se incrementa en el periodo o tiempo de almacenamiento (Novoa et al., 2006; Incotec, 1999). De esta manera, se encontró que durante los días de almacenamiento los mayores porcentajes se presentaron en el tratamiento no recubierto (tabla 4).

Tabla 3. Valores de $\mathrm{pH}$ en las gayabas muestreadas.

\begin{tabular}{|c|c|c|c|c|c|c|c|}
\hline \multirow{2}{*}{ Tratamientos } & \multicolumn{6}{|c|}{ Días } & \multirow{2}{*}{ Promedio } \\
\hline & 0 & 3 & 6 & 9 & 12 & 15 & \\
\hline T1 (A1B1) & 3.80 & 3.93 & 3.89 & 3.85 & 3.97 & 3.96 & 3.90 \\
\hline T2 (A1B2) & 3.65 & 3.91 & 3.90 & 3.98 & 4.08 & 3.99 & 3.92 \\
\hline T3 (A2B1) & 3.88 & 3.88 & 3.83 & 3.79 & 3.98 & 3.95 & 3.89 \\
\hline T4 (A2B2) & 3.85 & 3.82 & 3.82 & 3.86 & 3.84 & 3.89 & 3.85 \\
\hline T5 (A3B1) & 3.83 & 3.72 & 3.75 & 3.74 & 3.77 & 3.81 & 3.77 \\
\hline T6 (A3B2) & 3.77 & 3.81 & 3.74 & 3.80 & 3.87 & 3.92 & 3.82 \\
\hline T7 (Testigo) & 3.87 & 4.00 & 3.94 & 4.10 & 4.26 & 4.27 & 4.07 \\
\hline
\end{tabular}

Fuente: elaboración propia. 
Tabla 4. Sólidos solubles totales promedio (\%) de las guayabas muestreadas.

\begin{tabular}{|c|c|c|c|c|c|c|c|}
\hline \multirow{2}{*}{ Tratamientos } & \multicolumn{6}{|c|}{ Días } & \multirow{2}{*}{ Promedic } \\
\hline & 0 & 3 & 6 & 9 & 12 & 15 & \\
\hline $\mathrm{T} 1$ (A1B1) & 7.50 & 7.67 & 8.17 & 8.33 & 9.00 & 9.27 & 8.32 \\
\hline $\mathrm{T} 2$ (A1B2) & 8.27 & 7.67 & 8.17 & 8.33 & 8.57 & 9.33 & 8.39 \\
\hline T3 (A2B1) & 7.67 & 7.83 & 7.93 & 8.00 & 8.17 & 8.50 & 8.02 \\
\hline T4 (A2B2) & 8.33 & 7.60 & 7.83 & 9.00 & 9.17 & 9.40 & 8.56 \\
\hline T5 (A3B1) & 7.70 & 6.77 & 7.27 & 7.50 & 7.77 & 7.70 & 7.45 \\
\hline T6 (A3B2) & 7.67 & 7.17 & 7.33 & 8.20 & 8.53 & 8.67 & 7.93 \\
\hline T7 (Testigo) & 8.67 & 8.77 & 8.83 & 9.10 & 9.67 & 9.93 & 9.16 \\
\hline
\end{tabular}

Fuente: elaboración propia.

Dichos valores aumentaron de manera constante a medida que aumentó el tiempo almacenamiento; pero más en T7, el cual Ilegó a tener valores de hasta 9.93 promediando 9.16 durante todo el periodo de evaluación; a diferencia de los tratamientos con recubrimiento cuyos valores promedio estuvieron en el rango de 7.45 y 8.56 . El menor valor promedio se presentó en el T5 (7.45), resultados similares a los obtenidos por Saradhuldhat y Paull (2007).

En análisis de varianza muestra diferencia significativa para los tratamientos en estudio, demostrando la influencia del recubrimiento en los ${ }^{\circ}$ Brix, teniéndose que las mayores cantidades se observan en el testigo. El test de Tukey $(p<0.05)$ presenta como mejor tratamiento al T5 (7.45). La figura 3 presenta los sólidos solubles observándose a T7 con mayores valores y T5 con los menores, favoreciendo en el proceso de madurez.

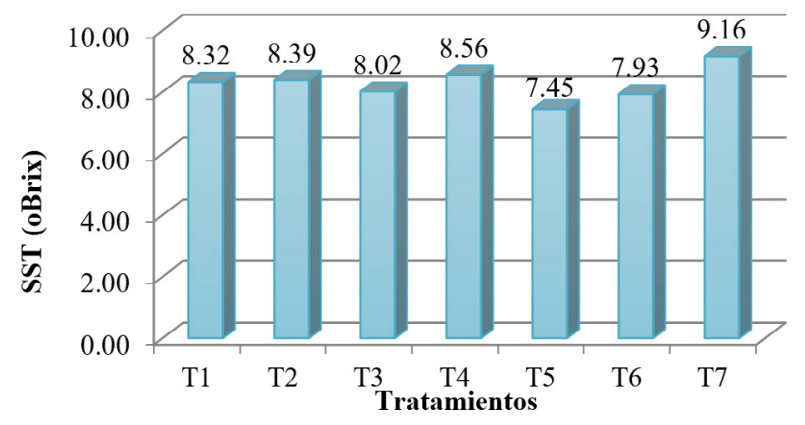

Figura 3. Sólidos solubles totales en frutos de guayaba durante el periodo de evaluación.

Fuente: elaboración propia.

\section{Acidez titulable}

Este parámetro debe su variación a la degradación de los ácidos orgánicos en el ciclo de Krebs en los primeros estados de la maduración, cuando la concentración de azúcares es baja y ocasiona la desaparición del sabor ácido del fruto (Saradhuldhat y Paull, 2007). La tabla 5 presenta las mediciones de acidez titulable en cada tratamiento y días de evaluación, así como también los promedios alcanzados. Conforme a estos, es posible observar que la acidez titulable baja con el tiempo de almacenamiento. Es importante indicar que este parámetro mantiene una correlación negativa con los sólidos solubles totales.

En el mismo contexto, se apreció que en el tratamiento sin recubrimiento (T7) el ácido cítrico llegó a disminuir hasta un valor promedio de 0.30 , comparado con los tratamientos con recubrimiento que se mantuvieron muy por encima de este; lo cual concuerda con investigaciones como la de Espinoza (2015) en recubrimiento sobre la calidad postcosecha de guayaba.

El análisis de varianza para la acidez titulable demuestra diferencias entre los tratamientos en estudio. Por otro lado, el test de Tukey al $0.05 \%$ selecciona a T5 como mejores tratamientos, ya que mantiene valores de 0.46 y T6 con igual concentración de Aloe vera con un $0.43 \%$. De la misma manera, se encuentra al tratamiento sin recubrimiento (T7) con el menor valor promedio durante 
el almacenamiento, lo cual indicaría que el proceso de senescencia se está desarrollando, tal como mencionan Saradhuldhat y Paull (2007) sobre la baja concentración de azucares y desaparición del ácido en la guayaba. Al respecto, la figura 4 se muestran los valores de cada tratamiento durante cada una de las evaluaciones durante el periodo de almacenamiento.

\section{Análisis microbiológico}

La actividad microbiológica es una de las principales causas del deterioro de los alimentos, lo cual conlleva a la pérdida de calidad de los mismos. En este sentido, se cuenta con la premisa de que a medida que el fruto madura, se produce un aumento progresivo de la presencia de microorganismos, sobre todo mohos y levaduras (Fleet, 1999). Debido a ello, existe un creciente interés en el uso de compuestos antibacterianos naturales como medio de conservación de alimentos (Acosta, 2014).

La tabla 6 muestra el conteo de unidades formadoras de colonias (UFC) luego del análisis microbiológico realizado a las guayabas (figura 5). Se puede observar lo expuesto concerniente al aumento de microorganismos en la medida en que avanza la maduración. Así mismo, se determinó también que T7 posee los valores más altos de este parámetro (4.63 UFC/mL). Por otro lado, con menor valor promedio (3.05 UFC/mL) se encuentra T5. Los resultados concuerdan con lo establecido por Appendini y Hotchkiss (2002), quienes

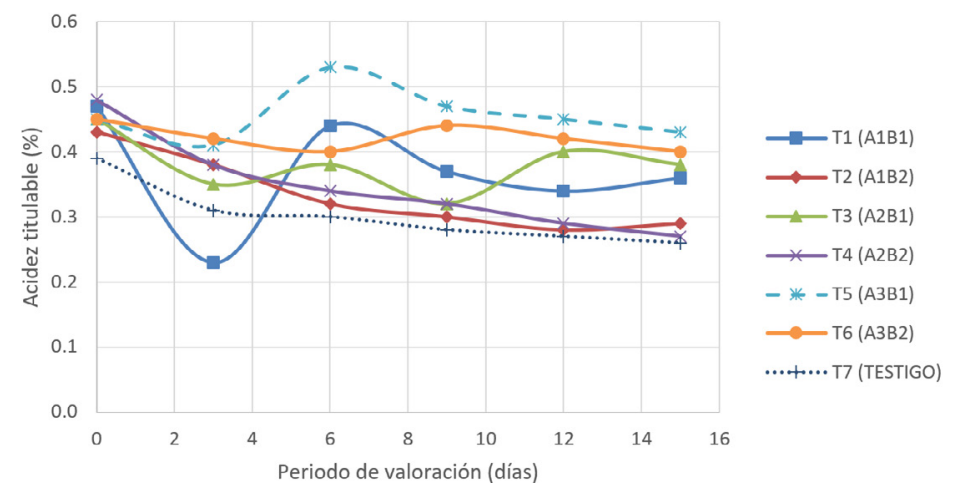

Figura 4. Acidez titulable en frutos de guayaba durante el periodo de evaluación.

Fuente: Elabpración propia.

Tabla 5. Acidez titulable en las gayabas muestreadas.

\begin{tabular}{|c|c|c|c|c|c|c|c|}
\hline \multirow{2}{*}{ Tratamientos } & \multicolumn{6}{|c|}{ Días } & \multirow{2}{*}{ Promedio } \\
\hline & 0 & 3 & 6 & 9 & 12 & 15 & \\
\hline T1 (A1B1) & 0.47 & 0.23 & 0.44 & 0.37 & 0.34 & 0.36 & 0.37 \\
\hline T2 (A1B2) & 0.43 & 0.38 & 0.32 & 0.30 & 0.28 & 0.29 & 0.33 \\
\hline T3 (A2B1) & 0.45 & 0.35 & 0.38 & 0.32 & 0.40 & 0.38 & 0.38 \\
\hline T4 (A2B2) & 0.48 & 0.38 & 0.34 & 0.32 & 0.29 & 0.27 & 0.35 \\
\hline T5 (A3B1) & 0.45 & 0.41 & 0.53 & 0.47 & 0.45 & 0.43 & 0.46 \\
\hline T6 (A3B2) & 0.45 & 0.42 & 0.40 & 0.44 & 0.42 & 0.40 & 0.42 \\
\hline T7 (Testigo) & 0.39 & 0.31 & 0.30 & 0.28 & 0.27 & 0.26 & 0.30 \\
\hline
\end{tabular}

Fuente: elaboración propia. 
mencionan que los recubrimientos, además de actuar como barrera de gases, pueden servir parara mejorar la seguridad de los alimentos mediante la inhibición en el crecimiento de microorganismo, dándole paso al concepto de envasado inteligente.

En este sentido, otras investigaciones referentes al recubrimiento en zanahorias (Durango et al., 2006), fresas (Mali y Grossmann, 2003) y frambuesas (Han et al., 2005) demuestran que indudablemente este método es viable en el control del crecimiento microbiano.

\section{Conclusiones}

El recubrimiento aplicado en guayaba con base en la sábila (Aloe vera) logra retardar el tiempo de maduración en la guayaba, conservándola y manteniendo a la fruta en buen estado a lo largo del tiempo de almacenamiento. Este recubrimiento logra obtener mejores resultados en cuanto a parámetros como: pérdida de peso, aumento del $\mathrm{pH}$, sólidos solubles, descenso de la acidez y conteo de mohos y levaduras, siendo eficiente para la conservación de la guayaba en comparación con las no recubiertas.

Mediante los monitoreos y análisis realizados, se establece que, en promedio, T5 fue el mejor tratamiento ya que perdió menos peso, mantiene un pH óptimo para la conservación, un bajo contenido de solidos solubles totales, el mayor contenido de ácido cítrico y menor conteo de unidades formadoras de colonias en el análisis microbiológico.

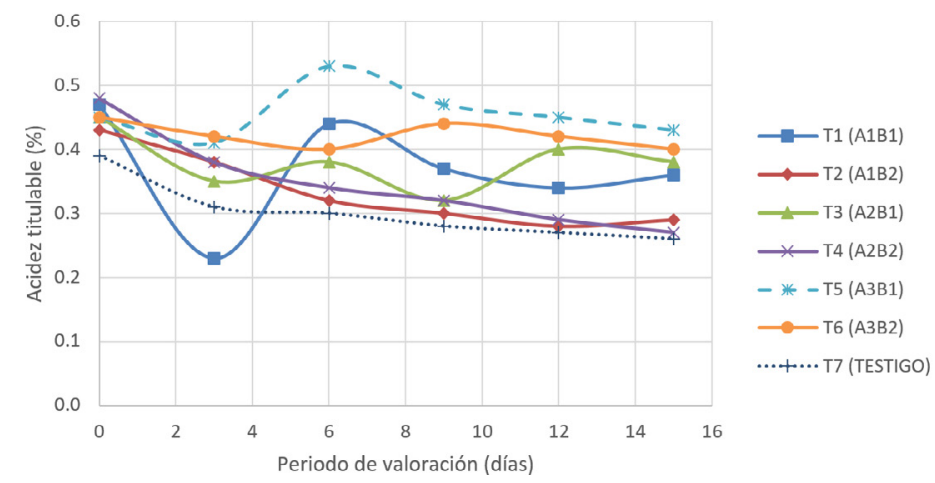

Figura 5. Resultados del análisis microbiológico de frutos de guayaba (UFC).

Fuente: Elaboración propia

Tabla 6. Presencia de mohos y levaduras (UFC/mL) en las gayabas muestreadas.

\begin{tabular}{cccccccc}
\hline & \multicolumn{7}{c}{ Días } \\
\cline { 2 - 6 } Tratamientos & 0 & 3 & 6 & 9 & 12 & 15 & \\
\cline { 2 - 6 } T1 (A1B1) & 2.80 & 3.00 & 3.60 & 3.80 & 4.00 & 4.00 & 3.53 \\
T2 (A1B2) & 2.80 & 3.50 & 3.80 & 4.00 & 4.40 & 4.50 & 3.83 \\
T3 (A2B1) & 2.80 & 3.10 & 3.50 & 3.90 & 4.20 & 4.80 & 3.72 \\
T4 (A2B2) & 2.80 & 3.30 & 4.33 & 4.20 & 4.50 & 5.00 & 4.02 \\
T5 (A3B1) & 2.80 & 2.90 & 3.10 & 3.10 & 3.10 & 3.30 & 3.05 \\
T6 (A3B2) & 2.80 & 3.00 & 3.90 & 4.00 & 4.20 & 4.30 & 3.70 \\
T7 (Testigo) & 2.80 & 3.50 & 4.20 & 4.80 & 6.00 & 6.50 & 4.63 \\
\hline
\end{tabular}

Fuente: Elabpración propia. 
De esta manera, se concluye que las guayabas Ilegaron a mantenerse hasta los 15 días de almacenamiento e inclusive se podría mantener por unos cinco días más.

Desde el punto de vista organoléptico, durante la realización de los análisis se podía observar que el recubrimiento lograba mantener las características apropiadas retardando que las guayabas maduren, manteniendo color, disminuyendo el aroma, manteniendo la textura, salvo el caso referente al sabor volviéndose un poco menos dulce.

\section{Referencias}

Achipiz, S., Castillo, A. E., Mosquera, S. A., Hoyos, J. L. y Navia, D. P. (2013). Efecto de recubrimiento a base de almidón sobre la maduración de la guayaba (Psidium guajava). Biotecnología en el sector agropecuario y agroindustrial, 11, 92-100.

Acosta, S. (2014). Propiedades de films de almidón de yuca y gelatina. ncorporación de aceites escenciales con efecto antifúngico (tesis de doctorado). Universidad Politécnica de Valencia, Valencia, España.

Appendini, P. y Hotchkiss, J. H. (2002). Review of antimicrobial food packaging. Innovative Food Science \& Emerging Technologies, 3(2), 113-126.

Agencia de Servicios a la Comercialización y Desarrollo de Mercados Agropecuarios (Aserca). (1996). Estudios del mercado mundial de la guayaba. Revista Claridades Agropecuarias, 41.

Bashir, H. A. y Abu-Goukh, A.-B. A. (2003). Compositional changes during guava fruit ripening. Food Chemistry, 80(4), 557-563.

Bastioli, C. (2001). Global status of the production of biobased packaging materials. Starch-Stärke, 53(8), 351-355.

Castellano, G., Quijada, O., Ramírez, R. y Sayago, E. (2005). Comportamiento poscosecha de frutas de guayaba (Psidium guajava $L$.) tratados con cloruro de calcio y agua caliente a dos temperaturas de almacenamiento. Revista Iberoamericana de Tecnología Postcosecha, 6(2), 78-82.

Cisternas, S. (1996). Uva de mesa (Vitis vinifera L.) cv. Red Globe: alternativas de control de Botrytis cinerea Pers. en postcosecha (tesis de maestría). Universidad de Chile, Santiago, Chile.

Cuq, B., Gontard, N. y Guilbert, S. (1998). Proteins as agricultural polymers for packaging production. Cereal Chemistry, 75(1), 1-9.

De la Cruz, G., Polo, M., Peniche, R. y Cárdenas, M. (1998). Efecto de la temperatura, humedad, relativa y tipo de recubrimiento sobre la repiración de la guayaba. CYTA-Journal of Food, 2(2), 54-59.

Durango, A. M., Soares, N. F. F. y Andrade, N. J. (2006). Microbiological evaluation of an edible antimicrobial coating on minimally processed carrots. Food Control, 17(5), 336-341.

Espinoza, N. (2015). Efecto de un recubrimiento comestible funcional a base de Goma Guar sobre la calidad poscosecha de guayaba (tesis). Universidad Autónoma Agraria Antonio Narro, Buenavista Saltillo, CoaHuila, México.

Famá, L., Goyanes, S. y Gerschenson, L. (2007). Influence of storage time at room temperature on the physicochemical properties of cassava starch films. Carbohydrate Polymers, 70(3), 265-273.

Fleet, G. H. (1999). Microorganisms in food ecosystems. International Journal of Food Microbiology, 50(1), 101-117.

Giovannoni, J. (2001). Molecular biology of fruit maturation and ripening. Annual Review of Plant Biology, 52(1), 725-749.

Gómez, S., Jurado, G. y Arcila Pulgarín, M. (2002). Comportamiento físico, químico y organoléptico de frutos de plátano dominico-hartón sometidos a diferentes sistemas de almacenamiento y tipos de empaques en el Quindio. Memorias XV Reunión Internacional ACORBAT. Cartagena de Indias, Colombia. 517-522 p. 
Gutiérrez, R., Pérez, M., Mitchell, S. y Vargas, R. (2008). Psidium guajava: a review of its traditional uses, phytochemistry and pharmacology. Journal of Ethnopharmacology, 117(1), 1-27.

Han, C., Lederer, C., McDaniel, M. y Zhao, Y. (2005). Sensory evaluation of fresh strawberries (Fragaria ananassa) coated with chitosanbased edible coatings. Journal of Food Science, 70(3), S172-S178.

Hintlian, C. B. y Hotchkiss, J. H. (1986). The safety of modified atmosphere packaging: a review. Food Technology, 40, 70-75.

Instituto Colombiano de Normas Técnicas y Certificación (Incotec). (1999). Frutas frescas. Uchuva. Especificaciones. Norma Técnica Colombiana NTC 4580. Bogotá, Colombia: Incotec.

Jagtian, J., Chan, H. y Sakai, W. (2012). Food Science and Technology. A series of Monograps. Tropical fruit processing. Academic Press, Inc. San Diego, California. 184 p.

Kader, A. A., Zagory, D., Kerbel, E. L. y Wang, C. Y. (1989). Modified atmosphere packaging of fruits and vegetables. Critical Reviews in Food Science \& Nutrition, 28(1), 1-30.

Lima, M., Coêlho De Assis, J. y Gonzaga, L. (2002). Caracterização dos frutos de goiabeira e seleção de cultivares na região do submédio São Francisco. Revista Brasileira de Fruticultura, 24(1), 273-276.

Mali, S. y Grossmann, M. (2003). Effects of yam starch films on storability and quality of fresh strawberries (Fragaria ananassa). Journal of Agricultural and Food Chemistry, 51(24), 7005-7011.

Mannapperuma, J., Zagory, D., Singh, R. y Kader, A. (1989). Design of polymeric packages for modified atmosphere storage of fresh produce. 5th International Controlled Atmosphere Research Conference.

Martínez, G., Augusto, J., Ramírez, M., De la Rosa, L. y Pozo, O. (2005). Efectos genéticos y heterosis en la vida de anaquel del chile serrano. Rev. Fitotec. Mex, 28(4), 327-332.
Ni, Y., Turner, D., Yates, K. M. y Tizard, I. (2004). Isolation and characterization of structural components of Aloe vera L. leaf pulp. International Immunopharmacology, 4(14), 1745-1755.

Novoa, R., Bojacá, M., Galvis, J. y Fischer, G. (2006). La madurez del fruto y el secado del cáliz influyen en el comportamiento poscosecha de la uchuva, almacenada a $12 \mathrm{C}$ (Physalis peruviana L.). Agronomía colombiana, 24(1), 77-86.

Oms-Oliu, G. et al. (2010). Recent approaches using chemical treatments to preserve quality of fresh-cut fruit: A review. Postharvest Biology and Technology, 57(3), 139-148.

Pech, J.-C., Bouzayen, M. y Latché, A. (2008). Climacteric fruit ripening: ethylene-dependent and independent regulation of ripening pathways in melon fruit. Plant Science, 175(1), 114-120.

Perez-Gago, M., Serra, M., Alonso, M., Mateos, M. y Del Río, M. A. (2005). Effect of whey protein-and hydroxypropyl methylcellulose-based edible composite coatings on color change of fresh-cut apples. Postharvest Biology and Technology, 36(1), 77-85.

Petersen, K., Nielsen, P. V., Bertelsen, G., Lawther, M., Olsen, M. B., Nilsson, N. H. y Mortensen, G. (1999). Potential of biobased materials for food packaging. Trends in Food Science \& Technology, 10(2), 52-68.

Possel, R. (1992). Tipos de embalaje y aplicación de difenilamina en la conservación de frutos de níspero Eriobotrya japonica Lindl.), cv."Golden Nugget" (memoria de titulo). Facultad de Ciencias Agrarias, Universidad de Chile, Santiago, Chile.

Quila, F. (2003). Efecto de tres temperaturas y tres períodos de almacenaje sobre el comportamiento en postcosecha de frutos de Níspero (Eriobotrya japonica, Lindl) cv. Golden Nugget. Taller Ing. Agr. Facultad de Agronomía, Pontificia Universidad Católica de Valparaíso, QuiIlota, Chile. 
Ramírez, J., Aristizábal, T. y Restrepo, J. (2013). Conservación de mora de castilla mediante la aplicación de un recubrimiento comestible de gel de mucilago de sábila. Vitae, 20(3), 172-183.

Saenz, M. V, Barquero, C. y Calvo, G. (1991). Efecto del empaque y la temperatura de almacenamiento sobre la vida poscosecha y la calidad de maracuya (Passiflora edulis var. flavicarpa). Agronomia Costarricense, 15, 79-83.

Salazar, D. M., Melgarejo, P., Martínez, R., Martínez, J. J., Hernández, F. y Burguera, M. (2006). Phenological stages of the guava tree (Psidium guajava L.). Scientia Horticulturae, 108(2), 157-161.

Sanabria, H. L., García, M. A., Díaz, H. A. y Muñoz, J. E. (2005). Caracterización morfológica en árboles nativos de guayaba en el Valle del Cauca. Acta Agronómica, 54(4), 1.

Saradhuldhat, P. y Paull, R. E. (2007). Pineapple organic acid metabolism and accumulation during fruit development. Scientia Horticulturae, 112(3), 297-303.

Sarroca, R. y Torres, M. (2006). Manipulación y almacenamiento de alimentos. Cuba: Centro de Investigación y Desarrollo del Comercio Interior y Sociedad Cubana de Logística y Marketing.
Sundaresan, K. V. y Ludescher, R. D. (2008). Molecular mobility and oxygen permeability in amorphous $\beta$-lactoglobulin films. Food Hydrocolloids, 22(3), 403-413.

Tomás, S. A., Bosquez-Molina, E., Stolik, S. y Sánchez, F. (2005). Effects of mesquite gum-candelilla wax based edible coatings on the quality of guava fruit (Psidium guajava L.). Journal de Physique IV, 125, 889-892. Doi: 10.1051/ jp4:2005125206

Vasco, N., Padilla, J. y Toro, J. (2003). Composición nutrimental de la guayaba y sus semillas. Primer Simposio Internacional de la Guayaba (pp. 116-123).

Yam Tzec, J., Perea, V., Alberto, C., Romantchik Kriuchkova, E., Soto, M. y Peña, M. A. (2010). Una revisión sobre la importancia del fruto de guayaba (Psidium guajava L.) y sus principales características en la postcosecha. Revista Ciencias Técnicas Agropecuarias, 19(4), 74-82.

Zebadúa, F., Vargas, L., González, S., Tamayo, J., Sauri, E. y Centurión, A. (2005). Efecto del empaque en bolsas de PBD sobre la calidad y vida útil de la pitahaya (Hylocereus undatus) durante su refrigeración. V Congreso Iberoamericano de Ingeniería de Aliemntos (Cibia V). Jaliso, México: 